\title{
A simple event weighting technique: optimizing the measurement of the forward-backward asymmetry of Drell-Yan dilepton and top-antitop pairs at hadron colliders
}

\author{
A. Bodek ${ }^{\mathrm{a}}$ \\ Department of Physics and Astronomy, University of Rochester, Rochester, NY 14627-0171, USA
}

Received: 26 November 2009 / Revised: 11 February 2010 / Published online: 23 March 2010

(C) Springer-Verlag / Società Italiana di Fisica 2010

\begin{abstract}
We describe a simple technique for optimizing the extraction of the forward-backward asymmetry $\left(A_{\mathrm{fb}}\right)$ of Drell-Yan lepton pairs $\left(e^{+} e^{-}, \mu^{+} \mu^{-}\right)$produced in $\bar{p} p$ and $p p$ collisions at hadron colliders. The method employs simple event weights which are functions of the rapidity and $|\cos \theta|$ decay angle of the lepton pair. It yields the best estimate of the acceptance corrected parton level $(\bar{q} q)$ forward backward asymmetry as a function of final state dilepton mass $\left(M_{\ell \ell}\right)$. Typically, when compared to the simple count method, the technique reduces the statistical errors by $20 \%$ for $\bar{p} p$, and $40 \%$ for $p p$ collisions, respectively. The method is equivalent to using the maximum likelihood method, but is much easier to implement. The technique can be used to search for new high mass and large width $Z$ ' bosons which may be best detected through the observation of deviations from the Standard Model expectation for the forwardbackward asymmetry. The technique can also be applied in the extraction of the foward-backward asymmetry in the production of top-antitop pairs.
\end{abstract}

\section{Introduction}

The Drell-Yan process in which $q \bar{q}$ annihilations form intermediate $\gamma^{*}$ or $Z\left(\gamma^{*} / Z\right)$ vector bosons decaying to lepton $\left(e^{+} e^{-}, \mu^{+} \mu^{-}\right)$pairs is particularly useful in searching for new interactions at large momentum transfers, $Q^{2}=M_{\ell \ell}^{2}$, where $M_{\ell \ell}$ is the invariant mass of the lepton pair. In leading order (LO) approximation, the momentum fractions $x_{1}$, $x_{2}$ carried by the initial state quarks and antiquarks in the proton and antiproton/proton, respectively, are related to the

a e-mail: bodek@pas.rochester.edu rapidity $|y|^{1}$ of the $\gamma^{*} / Z$ boson via the equation $x_{1,2}=$ $\left(M_{\ell \ell} / \sqrt{s}\right) e^{ \pm|y|}$, where $\sqrt{s}$ is the center of mass energy. Dilepton pairs produced at large $|y|$ originate from collisions in which one parton carries a large and the other a small momentum fraction $x$.

Drell-Yan lepton pairs which are produced in $q \bar{q}$ annihilations display a forward-backward asymmetry because of the interference between photon and $Z$ boson exchange [1-3]. This forward-backward asymmetry would be modified by new resonances (e.g. additional heavier $Z^{\prime}$ bosons [1-3]) or new interactions at large mass scales.

Although the mass limits from LEP [4, 5] and Tevatron $(\bar{p} p)$ experiments CDF [6-8] and DO [9] for a variety of $Z^{\prime}$ models are in the $0.6-0.8 \mathrm{TeV} / c^{2}$ range, the limits are much lower if the $Z^{\prime}$ width (typically $\Gamma_{Z^{\prime}} \approx 0.01 \cdot M_{Z^{\prime}}$ ) is increased to account for the possibility of additional decays modes to exotic fermions (which are predicted in $E_{6}$ models [10-13]), and/or supersymmetric particles. The limits are even lower if one includes the possibility of a more general model with enhanced couplings to the third generation.

Such a $Z^{\prime}$ (which has larger width e.g. $\Gamma_{Z^{\prime}}=0.1 M_{Z^{\prime}}$ ) would produce only a small signal in the dilepton mass spectrum because the total cross section is proportional to the square of the amplitude. However, the change in the forward-backward asymmetry which results from the interference with the standard model process is linearly proportional to the amplitude and would be observable as a change in the forward backward asymmetry. This change will occur around the mass of the $Z^{\prime}$ boson, and also in some mass range below and above the mass of the $Z^{\prime}$ boson [14]. This

\footnotetext{
${ }^{1}$ We define $(\theta, \phi, z)$, where $\theta$ is the polar angle relative to the quark direction (the $+z$ axis), and $\phi$ the azimuth. For a lepton $\left(e^{+}+e^{-}\right.$or $\mu^{+}+\mu^{-}$) pair with transverse momentum $P_{T}=P \sin \theta$, energy $E_{T}=$ $E \sin \theta$, the rapidity $y=\frac{1}{2} \ln \frac{E+P_{z}}{E-P_{z}}$. Here, $P$ and $P_{z}$ are the magnitude and $z$ component of the momentum, and $E$ is the energy of the lepton pair.
} 
point is illustrated in Fig. 1 which is taken from Reference [14]. The figure compares early $\left(120 \mathrm{pb}^{-1}\right)$ high mass DrellYan $d \sigma / d M_{\ell \ell}$ data from CDF and DO, and early $A_{\mathrm{FB}}$ data from CDF to Standard Model theoretical predictions and (as an example) to a prediction with an extra $E_{6}$ boson with $M_{Z^{\prime}}=350 \mathrm{GeV} / c^{2}$ and $\Gamma_{Z^{\prime}}=0.1 M_{Z^{\prime}}$, for $\phi=60^{\circ}$ (solid) and $\phi=173^{\circ}$ (dotted). As can be seen for this case, the signal in $A_{\mathrm{fb}}$ is larger than the signal in $d \sigma / d M_{\ell \ell}$. Since such new particles or new interactions may be best detected through the observation of deviations from the Standard Model expectation for the forward-backward asymmetry, it is useful to devise experimental techniques to measure the forward-backward asymmetry $\left(A_{\mathrm{fb}}\right)$ with the best possible
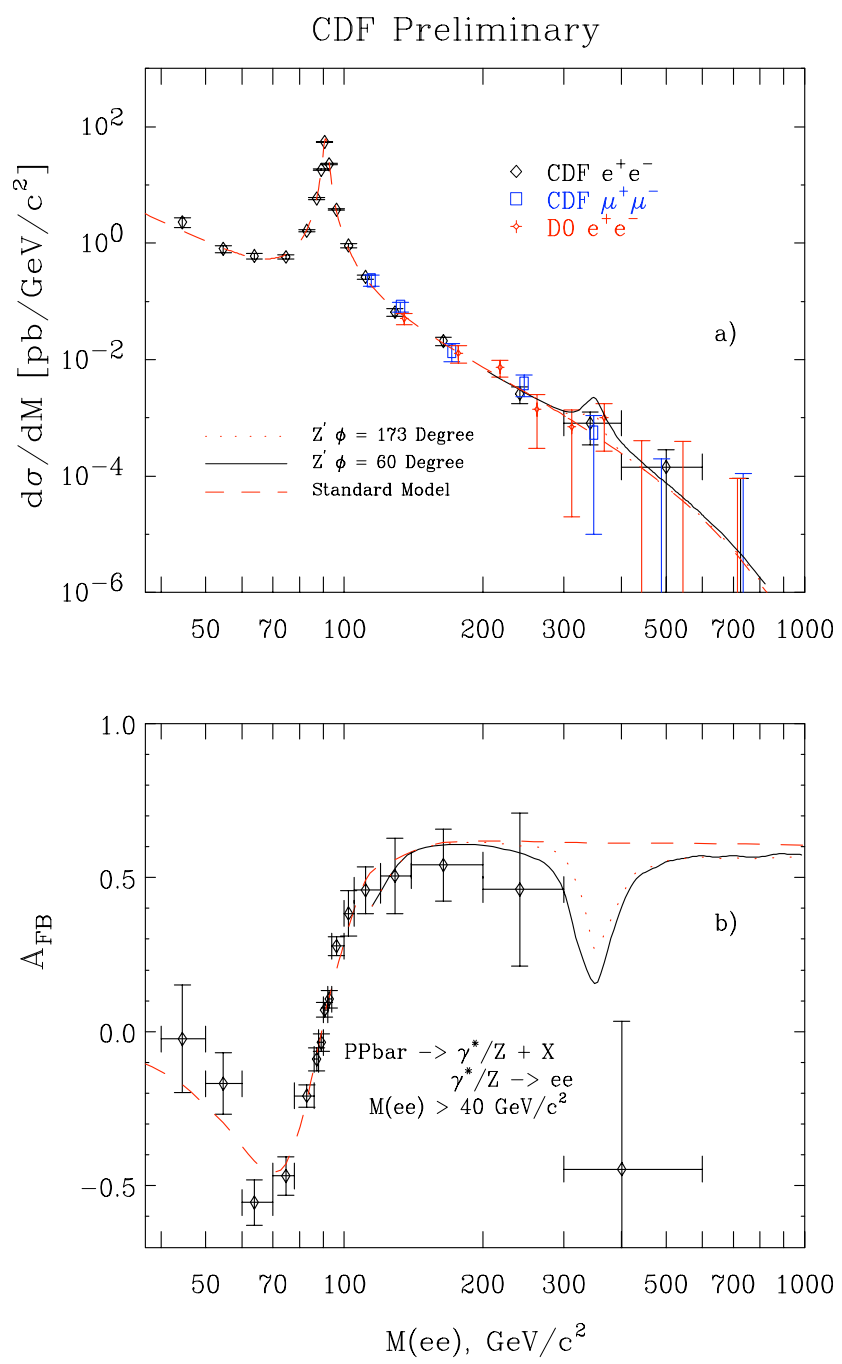

Fig. 1 Early Tevatron data $\left(120 \mathrm{pb}^{-1}\right)$. (a) $d \sigma / d M_{\ell \ell}$ for $e^{+} e^{-}$pairs from CDF and DO, and $\mu^{+} \mu^{-}$pairs from CDF. The Standard Model theoretical predictions (dashed line) have been normalized (by a factor of 1.11) to the CDF data in the $Z$ boson mass region. (b) CDF data for $A_{\mathrm{FB}}$ versus $M_{\ell \ell}\left(e^{+} e^{-}\right.$pairs). Also shown is the standard model expectation (dashed line) and the predictions for $d \sigma / d M$ and $A_{\mathrm{FB}}$ with an extra $E_{6}$ boson with $M_{Z^{\prime}}=350 \mathrm{GeV} / c^{2}$ and $\Gamma_{Z^{\prime}}=0.1 M_{Z^{\prime}}$, for $\phi=60^{\circ}$ (solid) and $\phi=173^{\circ}$ (dotted) precision. Since fine mass bins $\left(\approx 25 \mathrm{GeV} / c^{2}\right)$ are required, the number of events per bin at large $M_{\ell \ell}$ is small and the measurements are statistically limited.

In this communication we describe a simple technique for optimizing the extraction of the forward-backward asymmetry in the production of Drell-Yan dilepton pairs. The method employs simple event weights, which are functions of the rapidity and $|\cos \theta|$ decay angle of the lepton pair. The method yields the smallest statistical uncertainty in the measurement of the forward backward asymmetry as a function of $M_{\ell \ell}$. It can be directly applied to current $\bar{p} p$ data at the Fermilab Tevatron, as well as new data that will be collected in pp collisions at the Large Hadron Collider (LHC). The method works well for both large and small statistical samples. The method is equivalent to using the maximum likelihood method, but is much easier to implement. The technique can also be applied in the extraction of the fowardbackward asymmetry in the production of top-antitop pairs.

\section{$2 q \bar{q}$ annihilations}

The differential cross-section for the parton level process for $q \bar{q}$ annihilation can be written as

$\frac{d \sigma}{d(\cos \theta)}=A\left(1+\cos ^{2} \theta\right)+B^{\prime} \cos \theta$,

where $\theta$ is the emission angle of the positive lepton relative to the quark momentum in the center of mass frame, and $A$ and $B^{\prime}$ are parameters that depend on the weak isospin and charge of the incoming fermions. (We use $B^{\prime}$ for coefficient of the $\cos \theta$ at the parton level, and $B$ for the coefficient of the $\cos \theta$ at the nucleon level).

The cross sections for forward (for-) events $\left(\sigma_{F}\right)$ and backward (back-) events $\left(\sigma_{B}\right)$ are given by

$$
\begin{aligned}
\sigma_{f} & =\int_{0}^{1} \frac{d \sigma}{d(\cos \theta)} d(\cos \theta) \\
& =A\left(1+\frac{1}{3}\right)+B^{\prime}\left(\frac{1}{2}\right), \\
\sigma_{b} & =\int_{-1}^{0} \frac{d \sigma}{d(\cos \theta)} d(\cos \theta) \\
& =A\left(1+\frac{1}{3}\right)-B^{\prime}\left(\frac{1}{2}\right) .
\end{aligned}
$$

The electroweak interaction introduces the asymmetry (a linear dependence on $\cos \theta$ ), which can be expressed as

$A_{\mathrm{fb}}^{\mathrm{total}}=\frac{\sigma_{f}-\sigma_{b}}{\sigma_{b}+\sigma_{b}}=\frac{3 B^{\prime}}{8 A}$.

For $\bar{p} p$ collisions (e.g. at the Tevatron), the direction of the quark is predominately in the proton direction, and the 
direction of the antiquark is predominately in the antiproton direction. Therefore, the forward backward asymmetry for $q \bar{q}$ processes is easy to measure in $\bar{p} p$ collisions.

If $N_{f}$ is in number of events in the forward direction of the quark and $N_{b}$ is the number of events in the backward direction of the quark we obtain the following expression for the total forward backward-asymmetry $\left(A_{\mathrm{fb}}^{\text {total }}\right)$ and its error $\left(\Delta A_{\mathrm{fb}}^{\text {total }}\right)$ :

$$
\begin{aligned}
{\left[A_{\mathrm{fb}}\right]^{\text {total }} } & =\frac{N_{f}-N_{b}}{N_{f}+N_{b}}=\frac{N_{f}-N_{b}}{N}, \\
\frac{N_{f}}{N_{b}} & =\frac{1-A_{\mathrm{fb}}^{\text {total }}}{1+A_{\mathrm{fb}}^{\text {total }}}, \\
N_{f} & =\frac{1+A_{\mathrm{fb}}^{\text {total }}}{2} N, \\
N_{b} & =\frac{1-A_{\mathrm{fb}}^{\text {total }}}{2} N, \\
\Delta A_{\mathrm{fb}}^{\text {total }} & =\frac{2}{N}\left[\frac{N_{f} N_{b}}{N}\right]^{1 / 2}, \\
\Delta A_{\mathrm{fb}}^{\text {total }} & =\left[\frac{1-\left(A_{\mathrm{fb}(\text { expected })}^{\text {total }}\right)^{2}}{N}\right]^{1 / 2},
\end{aligned}
$$

where we have used $\Delta N_{f}=\left(N_{f}\right)^{1 / 2}$ and $\Delta N_{b}=\left(N_{b}\right)^{1 / 2}$, and $N=N_{f}+N_{b}$. Since for Poisson statistics [18, 19], the fractional error is $\left(1 / N_{\text {expected }}\right)^{1 / 2}$ and not $\left(1 / N_{\text {observed }}\right)^{1 / 2}$, we use $A_{\mathrm{fb} \text { (expected) }}$ in (6). For $\bar{p} p$ collisions above the $Z$ mass peak, $A_{\mathrm{fb}(\text { expected) }}=0.6$. In this region, $\Delta A_{\mathrm{fb}}=0.800$. $(1 / N)^{1 / 2}$.

Therefore, a measurement with 100 events yields a statistical error of 0.08 . This level of precision is needed to observe the deviation from the Standard Model for the $Z^{\prime}$ example shown in Fig. 1. Later in this paper we show that a reduction in the error (of about 20\%) can be obtained by using the information in the angular distribution of the forward and backward events.

\section{Quarks bound in a nucleon}

When quarks are bound in the nucleon, the dilepton can be produced with non-zero transverse momentum. This is described in detail in Appendix. For $p \bar{p}$ or $p p$ collisions the angular distribution of $\gamma^{*} / Z$ vector bosons decaying to $e^{+} e^{-}$or $\mu^{+} \mu^{-}$pairs is given by:

$$
\begin{gathered}
\frac{d \sigma}{d(\cos \theta)}=A\left[1+\cos ^{2} \theta+h(\theta)\right]+B \cos \theta, \\
h(\theta)=\frac{1}{2} A_{0}\left(M_{\ell \ell}, P_{T}\right)\left(1-3 \cos ^{2} \theta\right) .
\end{gathered}
$$

The $q \bar{q}$ center of mass frame is well defined when the lepton pair has zero transverse momentum $\left(P_{T}\right)$. For a non-zero transverse momentum of the dilepton pair, the $q \bar{q}$ center of mass frame is approximated by the Collins-Soper frame [15].

The term $h\left(\theta, M_{\ell \ell}, P_{T}\right)$ is a small QCD correction term which is zero when the transverse momentum of the dilepton pair is zero. As shown in Appendix, for quark-antiquark annihilation the angular coefficient $A_{0}$ is only a function of the dilepton mass $\left(M_{\ell \ell}\right)$ and transverse momentum $\left(P_{T}\right)$. The $h\left(\theta, M_{\ell \ell}, P_{T}\right)$ term integrates to zero when the cross section is integrated over all $\cos ^{2} \theta$. For simplicity we will use $A_{0}=0$ (e.g. $P_{T}=0$ ) in our calculations. In order to include the dependence on the $h\left(\theta, M_{\ell \ell}, P_{T}\right)$ term, we can use the following function derived in Appendix:

$A_{0}=\frac{P_{T}^{2} / M_{\ell \ell}^{2}}{1+P_{T}^{2} / M_{\ell \ell}^{2}}$.

QCD corrections also dilute the forward-backward asymmetry as follows:

$\frac{B}{B^{\prime}}=\frac{1}{\left(1+P_{T}^{2} / M_{\ell \ell}^{2}\right)^{1 / 2}}$.

On average, both QCD correction terms are very small. For Drell-Yan events the average ratio $P_{T} / M_{\ell \ell}$ is about 0.13 (e.g. $12 \mathrm{GeV} P_{T}$ at the $Z$ mass of $90 \mathrm{GeV}$ ). This implies that on average $\left\langle A_{0}\left(P_{T}\right)\right\rangle=0.018$, and $\left\langle\frac{B}{B^{\prime}}\left(P_{T}\right)\right\rangle=0.991$.

The emission of photons by the final state leptons also distorts the angular distribution (in addition to smearing events to lower mass). The effects of final state QED photons are best accounted for as part of an overall radiative correction. Alternatively, the angular distortion from QED radiation can also be corrected for by using effective angular coefficients which combine the QCD and QED corrections (expressions for the larger $A_{0}^{\mathrm{rad}}\left(P_{T}, M_{\ell \ell}\right)$ and larger $\frac{B^{\text {rad }}}{B^{\prime}}\left(P_{T}, M_{\ell \ell}\right)$ are given in Appendix $)$.

\section{Misidentification of the quark direction in $p p$ collisions}

For $\bar{p} p$ collisions the direction of the quark is primarily along the direction of the proton. However, there is a small probability for a misidentification (misID) that originates from the charge misidentification probability in the tracker. There is also an additional small misID that comes from the small fraction of events in which a sea antiquark in the proton interacts with a sea quark in the antiproton. This misID is very small for large dilepton final state mass.

We now turn to the measurement of the forward-backward asymmetry in $p p$ collisions (e.g. at the Large Hadron Collider). Although $p p$ collisions are symmetric, there is still a forward backward asymmetry if the quark direction is defined to be the direction of motion of the Drell-Yan pair. 
This originates from the fact that on average, quarks carry a larger fraction of the proton's momentum than antiquarks. However, there is a significant misID that originates from the fraction of events for which the antiquark carries a larger fraction of the momentum than the quark. This misID dilutes the observed asymmetry.

In most theoretical studies of the production of new $Z^{\prime}$ bosons in $p p$ collisions at the LHC, this dilution is included in calculations for the prediction for the observed forwardbackward asymmetry. Here, we show that we can obtain a higher sensitivity to new particle searches by correcting the data for the misID fraction on an event by event basis.

At small rapidity the misidentification probability $w_{i}$ is large $(0.5$ at $|y|=0)$. At large rapidity the misidentification probability $w_{i}$ is small. We show below that by taking this information into account we can reduce the error on the extracted $q \bar{q}$ asymmetry.

\section{Correcting for misID in $p p$ collisions}

We illustrate this point for the case of a high statistics measurement of the forward backward asymmetry in a specific mass bin (e.g $\left.450 \mathrm{GeV} / c^{2}\right)$.

We first extract the $q \bar{q}$ asymmetry by correcting the measured asymmetry for the average misID probability (this commonly used method is called the event count method).

We then show that we reduce the error on the extracted quark-antiquark asymmetry by binning the data in ten rapidity bins and fitting for the weighted average of the extracted parton level asymmetries from all of the ten rapidity bins.

Then we derive an event weighting technique that is equivalent to the fit method in the high statistics case, but which can also be used in the limit of very small statistical samples.

For proton-antiproton collisions we define the misID probability $\left(w_{i}\right)$ for each $y_{i}$ bin as the fraction of events for which the antiquark carries a larger fraction of the momentum than the quark. (For proton-antiproton collisions $w_{i}$ is the fraction of events for which the interactions occurs between a sea quark in the antiproton and a sea antiquark in the proton).

$x_{1(2)}=\frac{M_{\ell \ell}}{\sqrt{s}} \times e^{ \pm\left|y_{i}\right|}$,

$w_{i} \approx \frac{\sum_{\text {flavor }} v_{q}\left\{q\left(x_{2}\right) \cdot \bar{q}\left(x_{1}\right)\right\}}{\sum_{\text {flavor }} v_{q}\left\{q\left(x_{2}\right) \cdot \bar{q}\left(x_{1}\right)+q\left(x_{1}\right) \cdot \bar{q}\left(x_{2}\right)\right\}}$.

Where $M_{\ell \ell}$ is the dilepton mass $\left(M_{\mu \mu}\right.$ or $\left.M_{e e}\right)$, and $\left|y_{i}\right|$ is the rapidity of the dilepton pair. Here $q(x)$ denotes the quark distributions $(u(x), d(x), s(x), c(x), b(x))$ and $\bar{q}(x)$ denotes the antiquark distributions $(\bar{u}(x), \bar{d}(x), \bar{s}(x), \bar{c}(x), \bar{b}(x))$ for the various flavors in the nucleon. The parameter $v_{q}$ denotes the $Z / \gamma$ couplings of to each flavor (which a function of the dilepton mass). At large $M_{\ell \ell}$ the $u(x)$ and $\bar{u}(x)$ quark distributions dominate the expression for the dilution factor.

Note that the misID in the forward-backward asymmetry should include the interference between photon and $Z$ boson exchange for each quark flavor, which in general is a more complicated function of the couplings. In addition, the misID is affected by radiative emission of photons and detector resolution. Therefore, it is best to use a Monte Carlo generator (such as PYTHIA ${ }^{2}$ [16] or ZGRAD2 [17]) to empirically determine the functional dependence of the dilution factor $w_{i}$ as a function of the two measured variables $y_{\ell \ell \text {,measured }}$ and $M_{\ell \ell \text {,measured. }}$.

$w_{i}=f\left(y_{\ell \ell, \text { measured }}, M_{\ell \ell, \text { measured }}\right)$.

We now proceed to correct for the misID and extract the $q \bar{q}$ forward-backward asymmetry for each one of the ten $y_{i}$ rapidity bins. In the expressions below, $n_{f, i}$ and $n_{b, i}$ are the defined as the measured (i.e. diluted) number of forward events and backward events in each bin, and $N_{f, i}$ and $N_{b, i}$ are defined as the number of true forward and true backward events (for $q \bar{q}$ collisions) in the bin.

For a given $y_{i}$ rapidity bin with a misidentification probability $w_{i}$ the measured and true number of forward and backward events are related by the following expressions.

$n_{f, i}=N_{f, i}\left(1-w_{i}\right)+N_{b, i}\left(w_{i}\right)$,

$n_{b, i}=N_{b, i}\left(1-w_{i}\right)+N_{f, i}\left(w_{i}\right)$,

$N_{f, i}=n_{f, i}\left(1-w_{i}\right) / L_{i}-n_{b, i}\left(w_{i}\right) / L_{i}$,

$N_{b, i}=n_{b, i}\left(1-w_{i}\right) / L_{i}-n_{f, i}\left(w_{i}\right) / L_{i}$,

where $L_{i}=\left(1-2 w_{i}\right)$ is defined as the dilution factor. For a $\left|y_{i}\right|$ rapidity bin, the corrected parton level asymmetry is given by

$$
\begin{aligned}
A_{\mathrm{fb}-i}^{\mathrm{total}} & =\frac{N_{f, i}-N_{b, i}}{N_{f, i}+N_{b, i}}, \\
N_{f, i}-N_{b, i} & =n_{f, i} / L_{i}-n_{b, i} / L_{i}, \\
N_{f, i}+N_{b, i} & =n_{f, i}+n_{b, i}=n_{i}
\end{aligned}
$$

which yields

$$
A_{\mathrm{fb}, i}^{\mathrm{total}}=\frac{1}{L_{i}} \frac{n_{f, i}-n_{b, i}}{n_{i}}
$$

\footnotetext{
${ }^{2}$ We use the default (MSEL $\left.=11\right)$ LO matrix element $(Z+0$ jet $)$ with CTEQ5L PDFs and electroweak coupling $\sin ^{2} \theta_{W}=0.232$. The parton showering produces the boson $P_{T}$. The CDF EWK/TOP standard $W / Z P_{T}$ tuning parameters are: $\operatorname{MSTP}(91)=1, \operatorname{PARP}(91)=2.10$, $\operatorname{PARP}(93)=15$ for the low $P_{T}$ Gaussian smearing, with PART $(62)=$ 1.25 and PARP $(62)=0.2$ for the $P_{T}$ evolution in $7-25 \mathrm{GeV}$ region. The underlying event is included as Tune A. The QED parton showering uses the same machinery as QCD parton showering aside from coupling differences.
} 
$\left[\Delta A_{\mathrm{fb}-i}^{\mathrm{total}}\right]=\frac{1}{L_{i}} \frac{2}{n_{i}}\left[\frac{n_{f, i} n_{b, i}}{n_{i}}\right]^{1 / 2}$,

where we have used $\Delta n_{f, i}=\left(n_{f, i}\right)^{1 / 2}$ and $\Delta n_{b, i}=\left(n_{b, i}\right)^{1 / 2}$.

We find that a measurement of the $q \bar{q}$ asymmetry in the case where there is misID probability of $w_{i}$ results in an increase of the error in the extracted parton level asymmetry by a factor of $1 / L_{i}$ (which is equivalent to reducing the number of events by a factor of $\left.L_{i}^{2}=\left(1-2 w_{i}\right)^{2}\right)$.

If we want to combine different $\left|y_{i}\right|$ bins together, we need to weight the events by the inverse of the square of the statistical error in each bin. This is achieved by multiplying the expressions for $N_{f, i}$ and $N_{b, i}$ by $L_{i}^{2}$. Since this factor appears both in the numerator and denominator of the expression for $A_{\mathrm{fb}-i}^{\text {total }}$, it does not change the extracted value or error of the parton level asymmetry. However, when we combine $\left|y_{i}\right|$ bins together using event weighting, this factor accounts for the difference in statistical errors between the $\left|y_{i}\right|$ bins as follows.

$$
\begin{aligned}
k_{1, i} & =\left(1-w_{i}\right)\left(1-2 w_{i}\right), \\
k_{2, i} & =\left(w_{i}\right)\left(1-2 w_{i}\right), \\
N_{\text {total }} & =\sum_{\text {all-events }}[1], \\
S_{f} & =\sum_{\text {for-events }} k_{1, i}-\sum_{\text {back-events }} k_{2, i}, \\
{\left[\Delta S_{f}\right]^{2} } & =\sum_{\text {for-events }} k_{1, i}^{2}+\sum_{\text {back-events }} k_{2, i}^{2}, \\
S_{b} & =\sum_{\text {back-events }} k_{1, i}-\sum_{\text {for-events }} k_{2, i}, \\
{\left[\Delta S_{b}\right]^{2} } & =\sum_{\text {back-events }} k_{1, i}^{2}+\sum_{\text {for-events }} k_{2, i}^{2}, \\
A_{\mathrm{fb}}^{\text {total }} & =\frac{S_{f}-S_{b}}{S_{f}+S_{b}} .
\end{aligned}
$$

Now $\left[\Delta S_{f}\right]$ and $\left[\Delta S_{b}\right]$ are correlated with each other in a complicated way. In order to simplify the calculation of the error, we combine terms to isolate sums which are for forward events, and sums which are for backward events, separately as follows:

$$
\begin{aligned}
k_{A, i} & =k_{1, i}-k_{2, i}=\left(1-2 w_{i}\right)^{2}, \\
k_{B, i} & =k_{1, i}+k_{2, i}=\left(1-2 w_{i}\right), \\
N_{\text {total }} & =\sum_{\text {all-events }}[1], \\
A & =S_{f}+S_{b}=A_{1}+A_{2}, \\
B & =S_{f}-S_{b}=B_{1}-B_{2}, \\
A_{1} & =\sum_{\text {forward-events }} k_{A, i},
\end{aligned}
$$

$$
\begin{aligned}
A_{2} & =\sum_{\text {back-events }} k_{A, i}, \\
B_{1} & =\sum_{\text {forward-events }} k_{B, i}, \\
B_{2} & =\sum_{\text {back-events }} k_{B, i}, \\
{\left[\Delta A_{1}\right]^{2} } & =\sum_{\text {forward-events }} k_{A, i}^{2}, \\
{\left[\Delta A_{2}\right]^{2} } & =\sum_{\text {back-events }} k_{A, i}^{2}, \\
{\left[\Delta B_{1}\right]^{2} } & =\sum_{\text {forward-events }} k_{B, i}^{2}, \\
{\left[\Delta B_{2}\right]^{2} } & =\sum_{\text {back-events }} k_{B, i}^{2}, \\
A_{\mathrm{fb}}^{\text {total }} & =\frac{B}{A}=\frac{B_{1}-B_{2}}{A_{1}+A_{2}} .
\end{aligned}
$$

Now $\Delta A_{1}$ is $100 \%$ correlated with $\Delta B_{1}$ and $\Delta A_{2}$ is $100 \%$ correlated with $\Delta B_{2}$. We handle these correlations as follows.

$$
\begin{aligned}
\Delta A_{1} & =\Delta B_{1} \cdot \frac{A_{1}}{B_{1}}, \\
\Delta A_{2} & =\Delta B_{2} \cdot \frac{A_{2}}{B_{2}}, \\
{\left[\Delta A_{\mathrm{fb}}^{\mathrm{total}}\right]^{2} } & =\frac{1}{\left(A_{1}+A_{2}\right)^{4}}\left[E_{1}^{2}+E_{2}^{2}\right], \\
E_{1}^{2} & =\frac{\left[\Delta B_{1}\right]^{2}}{B_{1}^{2}}\left(A_{2} B_{1}+A_{1} B_{2}\right)^{2}, \\
E_{2}^{2} & =\frac{\left[\Delta B_{2}\right]^{2}}{B_{2}^{2}}\left(A_{2} B_{1}+A_{1} B_{2}\right)^{2} .
\end{aligned}
$$

A specific numerical example is shown in Table 1. Here we show the case of a measurement of $A_{\mathrm{fb}}$ in ten bins of $|y|$ for the range of $|y|$ between 0 and 2.0. For this study we assume that an asymmetry of $A_{\mathrm{fb}}=0.6$ is measured with 1000 events (for $0<|y|<2$ ) in $p p$ collisions at the LHC. We assume that the differential cross section is constant in $|y|$. The misID probability is assumed to be $w(|y|)=0.5(2-|y|) / 2$ which is a simple approximation to the misID values for a dilepton mass of $450 \mathrm{GeV} / c^{2}$ at the LHC. In each range in $|y|$ we compare the error in the extracted $\bar{p} p$ asymmetry from a simple count of events; the error extracted from a least square fit to the values extracted from each $\left|y_{i}\right|$ bin, and the error from the our event weighting formula. As expected, the error from the least square fit to the ten $\left|y_{i}\right|$ bins is the same as the error from our weighting formula. The error using a simple count is about $20 \%$ larger than the error using the event weighting scheme. The last column shows the error for the case of $\bar{p} p$ collisions (with a misID $=0$ ). 
Table 1 Proton-proton collisions: Numerical example of measuring $A_{\mathrm{fb}}$ in ten bins of $|y|$ for the range of $|y|$ between 0 and 2.0. For this study we assume an asymmetry $A_{\mathrm{fb}}=0.6$ which is measured with 1000 events (for $0<|y|<2$ ) in $p p$ collisions at the LHC. We assume that the differential cross section is constant in $|y|$. The misID probability is assumed to be $w(|y|)=0.5(2-|y|) / 2$ which is a simple approximation to the misID values for a dilepton mass of $450 \mathrm{GeV} / c^{2}$.
In each range in $|y|$ we compare the error in the extracted $\bar{q} q$ asymmetry from a simple count of events, the error using a least square fit to the values extracted for all $\left|y_{i}\right|$ bins, and the error from the proposed event weighting formula. The error using a simple count is about $20 \%$ larger than the error using the proposed event weighting scheme. The last column shows the error for the case of $\bar{p} p$ collisions (with a misID $=0$ )

\begin{tabular}{|c|c|c|c|c|c|c|}
\hline $\begin{array}{l}|y|-\text { range } \\
\text { range }\end{array}$ & $\begin{array}{l}N \\
\text { bins }\end{array}$ & $\begin{array}{l}\text { simple Count } \\
\text { Error } p p\end{array}$ & $\begin{array}{l}|y| \text { bins fit } \\
\text { Error } p p\end{array}$ & $\begin{array}{l}\text { Weights } \\
\text { Error } p p\end{array}$ & $\begin{array}{l}\text { Improv. } \\
\text { Factor } p p\end{array}$ & $\begin{array}{l}\bar{p} p \\
\text { Error }\end{array}$ \\
\hline $0-0.2$ & 1 & 1.999 & 1.9991 & 1.9991 & 1.00 & 0.087 \\
\hline $0-0.4$ & 2 & 0.706 & 0.6301 & 0.6298 & 1.12 & 0.061 \\
\hline $0-0.6$ & 3 & 0.383 & 0.3350 & 0.3346 & 1.14 & 0.050 \\
\hline $0-0.8$ & 4 & 0.248 & 0.2145 & 0.2141 & 1.15 & 0.043 \\
\hline $0-1.0$ & 5 & 0.177 & 0.1512 & 0.1511 & 1.16 & 0.039 \\
\hline $0-1.2$ & 6 & 0.134 & 0.1136 & 0.1131 & 1.17 & 0.035 \\
\hline $0-1.4$ & 7 & 0.106 & 0.0886 & 0.0882 & 1.18 & 0.033 \\
\hline $0-1.6$ & 8 & 0.086 & 0.0711 & 0.0706 & 1.21 & 0.031 \\
\hline $0-1.8$ & 9 & 0.071 & 0.0582 & 0.0578 & 1.23 & 0.029 \\
\hline $0-2.0$ & 10 & 0.060 & 0.0483 & 0.0479 & 1.26 & 0.027 \\
\hline
\end{tabular}

Our method is equivalent to combining different $\left|y_{i}\right|$ bins together by weighting the events by the inverse of the square of the "expected" statistical error in each bin. This has been done by multiplying the expressions for $N_{f, i}$ and $N_{b, i}$ by $L_{i}^{2}$. Weighting by a statistical error which is based on the "expected" number of events in a bin (instead of the measured number of events in a bin) is more correct, and is equivalent $[18,19]$ to using the maximum likelihood technique for combining bins. Therefore, our event weighting technique is equivalent to using a maximum likelihood technique, but is much easier to implement.

\section{Including information in the angular distribution in $\bar{p} p$ collisions}

We now investigate how much can be gained by looking at the asymmetry in bins of $\left|c_{j}\right|=\left|\cos \theta_{j}\right|$. We start with the case of $\bar{q} q$ collisions and divide the sample into ten bins in $\left|\cos \theta_{j}\right|$. The asymmetry as a function of $\left|c_{j}\right|$ bin is:

$$
\begin{aligned}
A_{\mathrm{fb}-j}\left(c_{j}\right) & =\frac{\sigma_{f}\left(\left|c_{j}\right|\right)-\sigma_{b}\left(\left|c_{j}\right|\right)}{\sigma_{f}\left(\mid c_{j}\right)\left|+\sigma_{b}\right|\left(c_{j} \mid\right)}, \\
A_{\mathrm{fb}-j}\left(c_{j}\right) & =\frac{N_{f, j}-N_{b, j}}{N_{f, j}-N_{b, j}} \\
& =\frac{B\left|c_{j}\right|}{A\left(1+c_{j}^{2}+h\left(M_{\ell \ell}, \theta, P_{T}\right)\right.} \\
& =A_{\mathrm{fb}-j}^{\mathrm{total}}\left[\frac{8\left|c_{j}\right|}{3\left(1+c_{j}^{2}+h(\theta)\right.}\right] .
\end{aligned}
$$

At $\left|c_{j}\right|=\left|\cos \theta_{j}\right|=0$, the measured asymmetry $A_{\mathrm{fb}-j}(0)=0$. At $\left|c_{j}\right|=\left|\cos \theta_{j}\right|=0.45$, the measured asymmetry $A_{\mathrm{fb}-j}(0.45)=A_{\mathrm{FB}}^{\text {total }}$. At $\left|c_{j}\right|=\left|\cos \theta_{j}\right|=1$, the asymmetry $A_{\mathrm{fb}}(1)=(4 / 3) A_{\mathrm{fb}}^{\text {total }}$. The measured asymmetry in each $\left|c_{j}\right|=\cos \theta_{j}$ bin can be related to the total (integrated over all $\cos \theta$ ) asymmetry. Therefore each $\left|\cos \theta_{j}\right|$ bin provides an independent measurement $A_{\mathrm{fb}-j}^{\text {total }}$ of the total asymmetry.

$$
\begin{aligned}
A_{\mathrm{fb}-j}^{\mathrm{total}} & =\frac{3}{8} \cdot \frac{N_{f, j}-N_{b, j}}{N_{f, j}+N_{b, j}} \cdot \frac{1}{M_{j}}, \\
\Delta A_{\mathrm{fb}-j}^{\mathrm{total}} & =\frac{3}{8 M_{j}} \frac{2}{N_{f, j}+N_{b, j}}\left[\frac{N_{f, j} N_{b, j}}{N_{f, j}+N_{b, j}}\right]^{1 / 2}, \\
M_{j} & =\frac{\left|c_{j}\right|}{\left(1+c_{j}^{2}+h\left(M_{\ell \ell}, \theta, P_{T}\right)\right)},
\end{aligned}
$$

where we have used $\Delta N_{f, j}=\left(N_{f, j}\right)^{1 / 2}$ and $\Delta N_{b, j}=$ $\left(N_{b, j}\right)^{1 / 2}$.

The above expression shows that for case in which we have same number of events in each of the $\left|\cos \theta_{j}\right|$ bins, the error in the extracted measurement of $A_{\mathrm{fb}-j}^{\mathrm{total}}$ from the data in a specific $\left|c_{j}\right|=\left|\cos \theta_{j}\right|$ bin is equal to the error of the measured asymmetry in the bin divided by a factor $M_{j}=\frac{\left|c_{j}\right|}{\left(1+c_{j}^{2}+h(\theta)\right)}$. This factor comes from the fact that the extracted total forward-backward asymmetry is more sensitive to events at large $\left|c_{j}\right|=\left|\cos \theta_{j}\right|$.

We now convert the procedure to event weight technique. We define $A_{\mathrm{fb}-j}^{\text {total }}=(3 / 8)\left(N_{A, j} / N_{B, j}\right)$.

$N_{f, j}=N_{A, j}\left(1+c_{j}^{2}+h(\theta)\right)+\left|c_{j}\right| N_{B, j}$, 
$N_{b, j}=N_{A, j}\left(1+c_{j}^{2}+h(\theta)\right)-\left|c_{j}\right| N_{B, j}$.

From which we get:

$N_{A, j}=\frac{N_{f, j}}{2\left(1+c_{j}^{2}+h\right)}+\frac{N_{b, j}}{2\left(1+c_{j}^{2}+h\right)}$,

$N_{B, j}=\frac{N_{f, j}}{2\left|c_{j}\right|}-\frac{N_{b, j}}{2\left|c_{j}\right|}$.

In order to properly weight events for different $\left|\cos \theta_{j}\right|$ bins by the inverse of the square of the error for each bin we multiply the above expressions by

$M_{j}^{2}=\frac{c_{j}^{2}}{\left(1+c_{j}^{2}+h\left(M_{\ell \ell}, \theta, P_{T}\right)\right)^{2}}$

and get:

$$
\begin{aligned}
z_{1, j} & =\frac{1}{2} \frac{c_{j}^{2}}{\left(1+c_{j}^{2}+h(\theta)\right)^{3}}, \\
z_{2, j} & =\frac{1}{2} \frac{\left|c_{j}\right|}{\left(1+c_{j}^{2}+h(\theta)\right)^{2}}, \\
A_{1} & =N_{f, j} \cdot\left(z_{1, j}\right), \\
A_{2} & =N_{b, j} \cdot\left(z_{1, j}\right), \\
B_{1} & =N_{f, j} \cdot\left(z_{2, j}\right), \\
B_{2} & =N_{b, j} \cdot\left(z_{2, j}\right), \\
{\left[\Delta A_{1}\right]^{2} } & =N_{f, j} \cdot z_{1, j}^{2}, \\
{\left[\Delta A_{2}\right]^{2} } & =N_{b, j} \cdot z_{1, j}^{2}, \\
{\left[\Delta B_{1}\right]^{2} } & =N_{f, j} \cdot z_{2, j}^{2}, \\
{\left[\Delta B_{2}\right]^{2} } & =N_{b, j} \cdot z_{2, j}^{2}, \\
A_{j} & =N_{f, j}\left(z_{1, j}\right)+N_{b, j}\left(z_{1, j}\right) \\
& =A_{1}+A_{2}, \\
B_{j} & =N_{f, j}\left(z_{2, j}\right)-N_{b, j}\left(z_{2, j}\right) \\
& =B_{1}-B_{2}, \\
A_{\mathrm{fb}-j}^{\mathrm{total}} & =\frac{3}{8} \frac{B_{j}}{A_{j}}=\frac{3}{8} \frac{B_{1}-B_{2}}{A_{1}+A_{2}} .
\end{aligned}
$$

Now $\Delta A_{1}$ is $100 \%$ correlated with $\Delta B_{1}$ and $\Delta A_{2}$ is $100 \%$ correlated with $\Delta B_{2}$. We handle these correlations as follows.

$$
\begin{aligned}
& \Delta A_{1}=\Delta B_{1} \cdot \frac{A_{1}}{B_{1}}, \\
& \Delta A_{2}=\Delta B_{2} \cdot \frac{A_{2}}{B_{2}},
\end{aligned}
$$

$\left[\Delta A_{\mathrm{fb}-j}^{\mathrm{total}}\right]^{2}=\left[\frac{3}{8}\right]^{2} \frac{1}{\left(A_{1}+A_{2}\right)^{4}}\left[E_{1}^{2}+E_{2}^{2}\right]$,

$$
\begin{aligned}
& E_{1}^{2}=\frac{\left[\Delta B_{1}\right]^{2}}{B_{1}^{2}}\left(A_{2} B_{1}+A_{1} B_{2}\right)^{2}, \\
& E_{2}^{2}=\frac{\left[\Delta B_{2}\right]^{2}}{B_{2}^{2}}\left(A_{2} B_{1}+A_{1} B_{2}\right)^{2} .
\end{aligned}
$$

Table 2 shows the results of a numerical example for an asymmetry $A_{\mathrm{fb}}=0.6$ measured with 1000 events (we assume $h=0)$. Here $A_{\mathrm{fb}}^{\text {total }}$ is measured in ten bins of $|\cos \theta|$. In each $\left|\cos \theta_{j}\right|$ bin we compare the error from the standard error formula, and the error from the event weighting formula. As expected, the two methods yield identical results.

In addition, we show a comparison of the average $A_{\mathrm{fb}}^{\text {total }}$ for the ten $\left|\cos \theta_{j}\right|$ bins calculated in three different ways. The error in the average extracted from a least square fit to the $10 A_{\mathrm{fb}-j}^{\mathrm{total}}$ values $(0.0196)$ is close to the error in the average determined from the weighted sum of all the events (0.0210). The error in $A_{\mathrm{fb}}^{\text {total }}$ from the weighted sum of all the events is $20 \%$ lower than the error of 0.0253 obtained from a simple count of all forward and backward events.

For the case of low statistics, we can use the event weighting technique to combine all the events at all value of $|\cos \theta|$ (we do not need to bin the events in $|\cos \theta|$ ). The following are the formulae to extract the best value and error from the entire range in $|\cos \theta|$ using the event weighting technique:

$$
\begin{aligned}
z_{1, j} & =\frac{1}{2} \frac{c_{j}^{2}}{\left(1+c_{j}^{2}+h\left(\theta, P_{T}\right)\right)^{3}}, \\
z_{2, j} & =\frac{1}{2} \frac{\left|c_{j}\right|}{\left(1+c_{j}^{2}+h\left(\theta, P_{T}\right)\right)^{2}}, \\
N_{\text {total }} & =\sum_{\text {all-events }}[1], \\
A_{1} & =\sum_{\text {forward-events }}\left[z_{1, j}\right], \\
A_{2} & =\sum_{\text {back-events }}\left[z_{1, j}\right], \\
B_{1} & =\sum_{\text {forward-events }}\left[z_{2, j}\right], \\
B_{2} & =\sum_{\text {back-events }}\left[z_{2, j}\right], \\
{\left[\Delta A_{1}\right]^{2} } & =\sum_{\text {forward-events }}\left[z_{1, j}^{2}\right], \\
{\left[\Delta A_{2}\right]^{2} } & =\sum_{\text {back-events }}\left[z_{1, j}^{2}\right], \\
{\left[\Delta B_{1}\right]^{2} } & =\sum_{\text {forward-events }}\left[z_{2, j}^{2}\right],
\end{aligned}
$$


Table 2 Proton-antiproton collisions: Numerical example for an asymmetry $A_{\mathrm{fb}}=0.6$ measured with 1000 events. Here $A_{\mathrm{fb}}^{\text {total }}$ is measured in ten bins of $|\cos \theta|$. In each $\left|\cos \theta_{j}\right|$ bin we compare the error from the standard error formula, and the error from the event weighting formula. As expected, the two yield identical results. In addition, we show a comparison of the average $A_{\mathrm{fb}}^{\text {total }}$ of all ten $\left|\cos \theta_{j}\right|$ bins calculated two different ways. The error in the average extracted from a least square fit to the $10 A_{\mathrm{fb}-j}^{\text {total }}$ values (0.0196) is close to the error in the average determined from the weighted sum of all the events (0.0210). The error in $A_{\mathrm{fb}}^{\text {total }}$ from the weighted sum of all the events is $20 \%$ lower than the error of 0.0253 obtained from a simple count of all forward and backward events

\begin{tabular}{|c|c|c|c|c|c|c|}
\hline $\begin{array}{l}\left|c_{j}\right|= \\
\left|\cos \theta_{j}\right|\end{array}$ & $n_{f}$ & $n_{b}$ & $\begin{array}{l}A_{\mathrm{fb}-j}\left(\left|c_{j}\right|\right) \\
\text { measured }\end{array}$ & $\begin{array}{l}A_{\mathrm{fb}-j}^{\text {total }} \\
\text { extracted }\end{array}$ & $\begin{array}{l}\Delta A_{\mathrm{fb}-j}^{\text {total }} \\
\left|\cos \theta_{j}\right| \text { bin }\end{array}$ & $\begin{array}{l}\Delta A_{\mathrm{fb}-j}^{\text {total }} \\
\text { from weights }\end{array}$ \\
\hline 0.05 & 41 & 34 & 0.080 & 0.60 & 0.864 & 0.864 \\
\hline 0.15 & 47 & 29 & 0.235 & 0.60 & 0.284 & 0.284 \\
\hline 0.25 & 55 & 25 & 0.376 & 0.60 & 0.165 & 0.165 \\
\hline 0.35 & 63 & 21 & 0.499 & 0.60 & 0.114 & 0.114 \\
\hline 0.45 & 72 & 18 & 0.599 & 0.60 & 0.085 & 0.085 \\
\hline 0.55 & 82 & 16 & 0.676 & 0.60 & 0.066 & 0.066 \\
\hline 0.65 & 92 & 14 & 0.731 & 0.60 & 0.054 & 0.054 \\
\hline 0.75 & 104 & 14 & 0.768 & 0.60 & 0.045 & 0.046 \\
\hline 0.85 & 116 & 14 & 0.790 & 0.60 & 0.041 & 0.041 \\
\hline 0.95 & 128 & 14 & 0.799 & 0.60 & 0.038 & 0.028 \\
\hline all & 800 & 200 & 0.6 & 0.60 & 0.0196 & 0.0210 \\
\hline all & 800 & 200 & 0.6 & 0.60 & simple-count & 0.0253 \\
\hline
\end{tabular}

Table 3 Proton-antiproton collisions: Numerical example of measuring $A_{\mathrm{fb}}$ for different acceptance ranges in $|\cos \theta|$. The example is for an asymmetry $A_{\mathrm{fb}}=0.6$ measured with a total of 1000 events (for all values of $|\cos \theta|$ ). For each $|\cos \theta|$ range we show the error in $A_{\mathrm{fb}}$ from a simple count, the error from the event weighting procedure, and the error from fitting bins in $|\cos \theta|$ (which is very similar to event weighting). Also shown is the improvement factor in the error when the event weighting procedure is used (versus a simple count). For a typical range of $|\cos \theta|$, using the simple weighting formula leads to more than $20 \%$ reduction in the error.

\begin{tabular}{llllll}
\hline $\begin{array}{l}|\cos \theta| \\
\text { range }\end{array}$ & $\begin{array}{l}N \\
\text { bins }\end{array}$ & $\begin{array}{l}\text { simple Count } \\
\text { Error }\end{array}$ & $\begin{array}{l}|\cos \theta| \text { bins } \\
\text { fit Error }\end{array}$ & $\begin{array}{l}\text { Event Weights } \\
\text { Error }\end{array}$ & $\begin{array}{l}\text { Improvement } \\
\text { factor }\end{array}$ \\
\hline $0-0.1$ & 1 & 0.8642 & 0.8642 & 0.8642 & 1.000 \\
$0-0.2$ & 2 & 0.3042 & 0.2796 & 0.2687 & 1.132 \\
$0-0.3$ & 3 & 0.1644 & 0.1410 & 0.1403 & 1.172 \\
$0-0.4$ & 4 & 0.1058 & 0.0884 & 0.0881 & 1.201 \\
$0-0.5$ & 5 & 0.0749 & 0.0611 & 0.0611 & 1.225 \\
$0-0.6$ & 6 & 0.0563 & 0.0459 & 0.0454 & 1.241 \\
$0-0.7$ & 7 & 0.0442 & 0.0346 & 0.0354 & 1.247 \\
$0-0.8$ & 8 & 0.0358 & 0.0277 & 0.0288 & 1.242 \\
$0-0.9$ & 9 & 0.0298 & 0.0230 & 0.0242 & 1.227 \\
$0-1.0$ & 10 & 0.0253 & 0.0196 & 0.0210 & 1.205 \\
\hline
\end{tabular}

$$
\begin{aligned}
{\left[\Delta B_{2}\right]^{2} } & =\sum_{\text {back-events }}\left[z_{2, j}^{2}\right], \\
A & =A_{1}+A_{2}, \\
B & =B_{1}-B_{2}, \\
{\left[A_{\mathrm{fb}}\right]^{\text {total }} } & =\frac{3}{8} \frac{B}{A}=\frac{3}{8} \frac{B_{1}-B_{2}}{A_{1}+A_{2}}, \\
\Delta A_{1} & =\Delta B_{1} \cdot \frac{A_{1}}{B_{1}},
\end{aligned}
$$

$$
\begin{aligned}
\Delta A_{2} & =\Delta B_{2} \cdot \frac{A_{2}}{B_{2}}, \\
{\left[\Delta A_{\mathrm{fb}}^{\mathrm{total}}\right]^{2} } & =\left[\frac{3}{8}\right]^{2} \frac{1}{\left(A_{1}+A_{2}\right)^{4}}\left[E_{1}^{2}+E_{2}^{2}\right], \\
E_{1}^{2} & =\frac{\left[\Delta B_{1}\right]^{2}}{B_{1}^{2}}\left(A_{2} B_{1}+A_{1} B_{2}\right)^{2}, \\
E_{2}^{2} & =\frac{\left[\Delta B_{2}\right]^{2}}{B_{2}^{2}}\left(A_{2} B_{1}+A_{1} B_{2}\right)^{2} .
\end{aligned}
$$


Note that since we add up the forward and backwards events in separate sums, the weighting factors $z_{1, j}$ and $z_{2, j}$ are functions of the absolute value $|\cos \theta|$.

Table 3 shows a numerical example of measuring $A_{\mathrm{fb}}$ for different acceptance ranges in $|\cos \theta|$ (we assume $h=0$ ). The example is for an asymmetry $A_{\mathrm{fb}}=0.6$ and an a sample of 1000 events (for $0<|\cos \theta|<1$ ). For each range of acceptance in $|\cos \theta|$ we show the error in $A_{\mathrm{fb}}$ from a simple count, the error from the event weighting procedure, and the error from fitting bins in $|\cos \theta|$ (which is very similar to event weighting). Also shown is the improvement factor in the error when the event weighting procedure is used (versus a simple count). For a typical range of acceptance in $|\cos \theta|$, using the simple weighting formula leads to more than $20 \%$ reduction in the error.

Note that when we use the angular distribution weights, the extracted $A_{\mathrm{fb}}^{\text {total }}$ is automatically corrected for the acceptance in $|\cos \theta|$ (since the acceptance cancels to first order). However, $N_{\text {total }}=\sum_{\text {all-events }}[1]$ is equal to the observed number of events and is not corrected for acceptance. For $N_{\text {total }}$, an acceptance correction is needed for the determination of $d \sigma / d M_{\ell \ell}$.

\section{Combining misID weighting and angular distribution weighting in $p p$ collisions}

In $p p$ collisions each event is can be characterized by a misID factor $w_{i}\left(\left|y_{i}\right|\right)$ which is related to the quark and antiquark distribution $\left(x_{1,2}=\left(M_{\ell \ell} / \sqrt{s}\right) e^{ \pm|y|}\right)$ at its value of $\left|y_{i}\right|$. In addition, each event has a measured value of $\left|c_{j}\right|=\left|\cos \theta_{j}\right|$. The expressions for combining events with different $\left|c_{j}\right|=\left|\cos \theta_{j}\right|$ and misID $w_{i}$ values are given by:

$$
\begin{aligned}
k_{A, i} & =k_{1, i}-k_{2, i}=\left(1-2 w_{i}\right)^{2}, \\
k_{B, i} & =k_{1, i}+k_{2, i}=\left(1-2 w_{i}\right), \\
N_{\text {total }} & =\sum_{\text {all-events }}[1], \\
z_{1, j} & =\frac{1}{2} \frac{c_{j}^{2}}{\left(1+c_{j}^{2}+h(\theta)\right)^{3}}, \\
z_{2, j} & =\frac{1}{2} \frac{\left|c_{j}\right|}{\left(1+c_{j}^{2}+h(\theta)\right)^{2}}, \\
A_{1} & =\sum_{\text {for-events }}\left[z_{1, j} k_{A, j}\right], \\
A_{2} & =\sum_{\text {back-events }}\left[z_{1, j} k_{A, j}\right], \\
B_{1} & =\sum_{\text {for-events }}\left[z_{2, j} k_{B, j}\right],
\end{aligned}
$$

$$
\begin{aligned}
B_{2} & =\sum_{\text {back-events }}\left[z_{2, j} k_{B, j}\right], \\
{\left[\Delta A_{1}\right]^{2} } & =\sum_{\text {for-events }}\left[z_{1, j}^{2} k_{A, j}^{2}\right], \\
{\left[\Delta A_{2}\right]^{2} } & =\sum_{\text {back-events }}\left[z_{1, j}^{2} k_{A, j}^{2}\right], \\
{\left[\Delta B_{1}\right]^{2} } & =\sum_{\text {for-events }}\left[z_{2, j}^{2} k_{B, j}^{2}\right], \\
{\left[\Delta B_{2}\right]^{2} } & =\sum_{\text {back-events }}\left[z_{2, j}^{2} k_{B, j}^{2}\right], \\
A & =A_{1}+A_{2}, \\
B & =B_{1}-B_{2}, \\
{\left[\Delta A_{1}\right] } & =\left[\Delta B_{1}\right] \cdot \frac{A_{1}}{B_{1}}, \\
{\left[\Delta A_{2}\right] } & =\left[\Delta B_{2}\right] \cdot \frac{A_{2}}{B_{2}}, \\
A_{\mathrm{fb}}^{\text {total }} & =\frac{3}{8} \frac{B}{A}=\frac{3}{8} \frac{B_{1}-B_{2}}{A_{1}+A_{2}}, \\
{\left[\Delta A_{\mathrm{fb}}^{\text {total }}\right]^{2} } & =\left[\frac{3}{8}\right]^{2} \frac{1}{\left(A_{1}+A_{2}\right)^{4}}\left[E_{1}^{2}+E_{2}^{2}\right], \\
E_{1}^{2} & =\frac{\left[\Delta B_{1}\right]^{2}}{B_{1}^{2}}\left(A_{2} B_{1}+A_{1} B_{2}\right)^{2}, \\
E_{2}^{2} & =\frac{\left[\Delta B_{2}\right]^{2}}{B_{2}^{2}}\left(A_{2} B_{1}+A_{1} B_{2}\right)^{2} .
\end{aligned}
$$

Table 4 shows a numerical example of the improvement in the errors that can be realized by using the information for both the misID $\left(w_{i}\right)$ and $\left|\cos \theta_{i}\right|$ on an event by event basis, versus the measurement of $A_{\mathrm{fb}}$ using a simple count of all events for all values of $|\cos \theta|$ and $|y|$ (within the experimental acceptance). We show the case for an asymmetry $A_{\mathrm{fb}}=0.6$ measured with $10^{6} \mathrm{pp}$ events (over all values of $|\cos \theta|$ and $0<|y|<2$ ) for the case of a dilepton mass of $450 \mathrm{GeV} / c^{2}$ at the LHC. The two dimensional table shows the improvement factor in the errors (over the simple count method) when we use event weighting in both $|\cos \theta|$ and MisID as a function of $|y|$. For a typical experimental acceptance in $|\cos \theta|$ and $|y|$, the weighting formula leads to a $40 \%$ reduction in the error (over the error obtained from a simple count).

The following are the advantages for using the event weighting.

1. For $p p$ or $\bar{p} p$ collisions, if event weighting is done only in $|\cos \theta|$, the error in the asymmetry is typically reduced by a factor of 1.2. This is the method that should be used to extract the quark couplings from the average integrated asymmetry over all values of $|y|$. 
Table 4 Proton-proton collisions: numerical example of measuring $A_{\mathrm{fb}}$ integrating over various ranges of $|\cos \theta|$ and various ranges of $|y|$ for the case of a dilepton mass of $450 \mathrm{GeV} / c^{2}$ at the LHC. Here we assume that the asymmetry $A_{\mathrm{fb}}=0.6$ is measured with $10^{6}$ events for values of $|\cos \theta|$ and $0<|y|<2$. Shown is the improvement factor in the error (over the simple count method) when we use event weighting in both $|\cos \theta|$ and $w=$ MisID as a function of $|y|$. For a typical range of acceptance in $|\cos \theta|$ and $|y|$, the weighting formula leads to a $40 \%$ reduction in the error (over the error obtained from a simple count)

\begin{tabular}{|c|c|c|c|c|c|c|c|c|c|c|}
\hline$y$ - range & $0-0.2$ & $0-0.4$ & $0-0.6$ & $0-0.8$ & $0-1.0$ & $0-1.2$ & $0-1.4$ & $0-1.6$ & $0-1.8$ & $0-2.0$ \\
\hline \multicolumn{11}{|l|}{$\begin{array}{l}|\cos \theta| \\
\text { range }\end{array}$} \\
\hline $0-0.1$ & 1.00 & 1.12 & 1.14 & 1.15 & 1.16 & 1.17 & 1.18 & 1.21 & 1.23 & 1.26 \\
\hline $0-0.2$ & 1.13 & 1.27 & 1.29 & 1.30 & 1.31 & 1.32 & 1.34 & 1.37 & 1.39 & 1.43 \\
\hline $0-0.3$ & 1.17 & 1.31 & 1.34 & 1.35 & 1.36 & 1.37 & 1.38 & 1.42 & 1.44 & 1.48 \\
\hline $0-0.4$ & 1.20 & 1.35 & 1.37 & 1.38 & 1.39 & 1.41 & 1.42 & 1.45 & 1.48 & 1.51 \\
\hline $0-0.5$ & 1.23 & 1.37 & 1.40 & 1.41 & 1.42 & 1.43 & 1.45 & 1.48 & 1.51 & 1.54 \\
\hline $0-0.6$ & 1.24 & 1.39 & 1.41 & 1.43 & 1.44 & 1.45 & 1.46 & 1.50 & 1.53 & 1.56 \\
\hline $0-0.7$ & 1.25 & 1.40 & 1.42 & 1.43 & 1.45 & 1.46 & 1.47 & 1.51 & 1.53 & 1.57 \\
\hline $0-0.8$ & 1.24 & 1.39 & 1.42 & 1.43 & 1.44 & 1.45 & 1.47 & 1.50 & 1.53 & 1.56 \\
\hline $0-0.9$ & 1.23 & 1.37 & 1.40 & 1.41 & 1.42 & 1.44 & 1.45 & 1.49 & 1.51 & 1.55 \\
\hline $0-1.0$ & 1.21 & 1.35 & 1.37 & 1.39 & 1.40 & 1.41 & 1.42 & 1.46 & 1.48 & 1.52 \\
\hline
\end{tabular}

2. For $p p$ collisions, if the event weighting is done in both $|\cos \theta|$ and $|y|$, the error in the asymmetry is reduced by a factor of 1.4. For $\bar{p} p$ collisions if the weighting is done in both $|\cos \theta|$ and $|y|$, there is also a further reduction in the error.

3. For $p p$ or $\bar{p} p$ collisions if event weighting is done only in $|\cos \theta|$ the method provides the acceptance corrected asymmetry without applying any acceptance corrections for missing coverage in $|\cos \theta|$. The $|\cos \theta|$ acceptance fully cancels to first order. For proton-antiproton collisions, this is only true if the acceptance is the same for positive and negative values of $|\cos \theta|$. If it is not, then the small difference in the acceptance for positive and negative $|\cos \theta|$ (e.g. dead channels) needs to be accounted for in a MC acceptance correction. Effects like a displaced beam vertex need to be corrected for using a MC simulation (for the proton-antiproton case). However, for proton-proton collisions, effects of the differences in the detector between forward and backward hemispheres or a displaced beam spot completely cancel. This is because for proton-proton collisions the forwardbackwards are defined with respect to the rapidity of the dilepton, which can be either positive or negative in the detector. A correction for the acceptance in $|y|$ still needs to applied in order to extract the average integrated asymmetry over all values of $|y|$. This is the method that should be used to extract the quark couplings from the average integrated asymmetry over all values of $|y|$.

4. If the event weighting is be done in both $|\cos \theta|$ and $|y|$ then both the $|\cos \theta|$ acceptance and the $|y|$ acceptance fully cancel. This is the method that should be used in a search for new $Z^{\prime}$ bosons. It should also be used for the extraction of the asymmetry in the production of top-antitop pairs, for which the acceptance is a complicated function of both $|\cos \theta|$ and $|y|$.

5. Only small corrections need to be made for detector resolution and radiative smearing effects. Most of these correction are already included if an empirical fit $w_{i}=$ $f\left(y_{\ell \ell, \text { measured }}, M_{\ell \ell, \text { measured }}\right)$ is used.

6. Only very small corrections need to be made for QCD modification of the angular distribution. Most of these corrections are already included if we use an empirical fit for $h\left(\theta, M_{\ell \ell}, P_{T}\right)$, as given in Appendix.

7. The method uses event weights which are functions of either $|y|$ or $|\cos \theta|$. This is equivalent to weighting the data (in bins in $|y|$ or $|\cos \theta|$ ) by the error in the expected number of events. Therefore, this is equivalent $[18,19]$ to using the maximum likelihood method (which yields the smallest error). However, the implementation of this technique is much easier and does not depend on a detailed modeling of the detector.

8. If the event weighting is be done in both $|\cos \theta|$ and $|y|$ then both the $|\cos \theta|$ acceptance and the $|y|$ acceptance cancel. Therefore, for this case the weighted sums for $A_{1}, A_{2}, B_{1}, B_{2}, \Delta B_{1}$, and $\Delta B_{2}$ from different run conditions, or different final state leptons (muon, electrons) or different experiments (e.g. Dzero and CDF or CMS and ATLAS) can be directly added to provide a combined result. This is important when there are only a few events in each detector (e.g. Drell-Yan events at high mass or top-antitop events). 


\section{Correction factors and systematic uncertainties in the weighting procedure}

For $p p$ collisions, the acceptance for forward and backward events is equal because of symmetry. Therefore the functional dependence of the acceptance in $|\cos \theta|$ fully cancels (if the acceptance for positive and negative muons is the same). For $\bar{p} p$ collisions one needs to correct for a possible small difference in the detector performance between the proton and antiproton directions. If the event weighting is be done in both $|\cos \theta|$ and $|y|$ then both the $|\cos \theta|$ acceptance and the $|y|$ acceptance cancel.

\subsection{Backgrounds and systematic errors}

The main experimental background is QCD dijet events. The QCD jet background is measured by statistically separating isolated muons (or electrons) from muons (or electron like objects) in jets on the basis of the transverse energy profile distributions in the calorimeter [20] (e.g. isolation energy variables).

In general QCD processes are mediated via spin 1 gluon exchange and therefore have the same angular distribution as Drell Yan events. If such is the case, the fractional QCD background is the same at all values of $|\cos \theta|$ and is the same for positive and negative values of $|\cos \theta|$. Therefore, in the expressions for the asymmetry, the QCD background cancels in the numerator. Corrections for this background only increases the level of the denominator. Therefore, a single multiplicative factor equal to $1 /(1-f)$ (where $f$ is the fraction of QCD background events) can be used to correct the extracted $A_{\mathrm{fb}}$ for QCD background. The same multiplicative correction factor for QCD background can be use for all three methods (simple count, fit to bins in $|\cos \theta|$ and event weighting technique). Possible deviations from this assumption can be included in the systematic error.

Another background originates from electroweak (EW) processes $\left(W W, W Z, W+\right.$ jets, $\tau^{+} \tau^{-}$, and $\left.t \bar{t}\right)$. This background is generally estimated from a Monte Carlo simulation. If the event weighting technique is used to extract the forward-backward asymmetry, then the event weighting technique can be used on Monte Carlo samples for the Drell-Yan signal and the electroweak background processes to determine the shift in $A_{\text {fb }}$ from EW backgrounds. Note that the contribution from $\tau^{+} \tau^{-}$events is very small (and at high mass $A_{\mathrm{fb}}$ for $\tau^{+} \tau^{-}$is similar to $A_{\mathrm{fb}}$ for $\mu \mu$ and $e^{+} e^{-}$ events).

As is generally the case in particle physics experiments, the procedure needs to be tested on Monte Carlo simulated data to determine the size of any pulls from the previously listed systematic effects. We note that this process to determine biases and pulls needs to be done for any procedure that is used to extract the forward-backward asymmetry for $q \bar{q}$ processes from data. Our procedure is designed to minimize these biases, but they still need to be determined from a full scale Monte Carlo simulation.

In $p p$ collisions at the LHC, there are also uncertainties from antiquark distribution functions that affect the misID probabilities (which are determined using a Monte Carlo simulations). The PDF uncertainties are smaller than the statistical errors for large dilepton mass. Therefore, this method is best applied to searches for new resonances or contact interactions at large mass. At large mass the leading order $\bar{q} q$ diagram dominates and the signal for new physics is local change in the asymmetry as a function of dilepton mass.

However, for precision measurement of Standard Model parameters at the LHC (e.g. near the region of the $Z$ boson) only weighting in $|\cos \theta|$ should be used. This is because at lower dilepton mass, gluon-quark processes are a significant fraction of the cross section, and the dilution correction is much more sensitive to PDFs and higher order QCD terms. At low mass, it is better to compare the measured asymmetry (using $|\cos \theta|$ weighting) to a complete NLO or NNLO QCD prediction.

\section{Summary}

We have shown that a simple event weighting technique can be used to reduce the statistical error on the extracted $\bar{q} q$ forward-backward asymmetry from Drell-Yan events in $\bar{p} p$ and $p p$ collisions. In addition to reducing the statistical error, the event weighting technique is much less sensitive to details of the experimental acceptance.

The asymmetry data for a dilepton mass above $300 \mathrm{GeV} / c^{2}$ (for both $p p$ and $\bar{p} p$ collisions) can be used to search for new $Z^{\prime}$ bosons.

\section{Appendix: Derivation of the perturbative formula for the angular coefficients}

We now show that for the $q \bar{q} \rightarrow \gamma^{*} / Z \rightarrow \ell^{+} \ell^{-}$annihilation diagram the perturbative calculation indicates that the angular coefficients originates from a kinematic effect which is a simple function of the angle of the proton and antiproton beams in the Collins-Soper frame. That angle is also a simple function of the transverse momentum $P_{T}$ and mass $M_{\ell \ell}$ of the dilepton pair.

We start with the case of the emission of a single hard gluon by one of the partons. In this case, the dilepton has a finite value of $P_{T}$ but we cannot determine if the gluon was emitted by the parton in the proton, or by the parton in the antiproton. All we know is that the dilepton pair has a finite transverse momentum in the laboratory system which is equal to the transverse momentum of the gluon. 
If the gluon is emitted by the parton in the antiproton, then the parton level angular distribution (given below in $\cos \theta^{\prime}$ ) is with respect to the direction of the proton (which defines the quark-antiquark axis. If the gluon is emitted by the parton in the proton, then the parton level angular distribution (given below in $\cos \theta^{\prime}$ ) is with respect to the direction of the antiproton (which defines the quark-antiquark axis). The Collins-Soper frame splits this difference and takes the average direction (which happens to be the same as the laboratory $z$ axis for colliding beam experiments).

In the derivation below, all vectors are defined in the Collins-Soper frame. We define unit vector $\mathbf{k}$ along the $z$ axis, unit vector $\mathbf{i}$ along the $x$ axis of the Collins-Soper frame (which is defined to be along the initial direction of the transverse momentum of the dilepton pair in the laboratory system). We also define a perpendicular unit vector $\mathbf{j}$ along the $y$ axis. Here $\theta$ is the angle with respect to the $z$ axis, and $\phi$ is defined in the $x-y$ plane.

In the Collins-Soper frame, the Drell-Yan pair is stationary and proton and antiproton move towards the dilepton pair with an angle $\xi$ with respect to the $z$ axis. In the CollinsSoper, we see an antiproton moving from the left at an angle $\xi$ with respect to the $z$ axis (in the $x-z$ plane), and a proton moving from the right with an angle $-\xi$ with respect to the $z$ axis. The proton and antiproton form an isosceles triangle with the two beam particles (which have equal momentum $p$ ) intersecting at an angle $2 \alpha=\pi-2 \xi$. Here $\cos \alpha=\sin \xi$. It can be easily shown that

$$
\begin{aligned}
\sin ^{2} \xi & =\frac{P_{T}^{2}}{P_{T}^{2}+M_{\ell \ell}^{2}}, \\
\cos \xi & =\frac{M_{\ell \ell}}{\left(P_{T}^{2}+M_{\ell \ell}^{2}\right)^{1 / 2}} .
\end{aligned}
$$

In the Collins-Soper frame, we define a unit vector $\mathbf{Z}^{\prime}$ along the direction of one of the beam particles (e.g. the proton). We define a unit vector $\mathbf{r}$ along the direction of Drell-Yan pair decay positron. We then obtain the following relations.

$$
\begin{aligned}
\mathbf{Z}^{\prime} & =\cos \xi \mathbf{k}+\sin \xi \mathbf{i}, \\
\mathbf{r} & =\sin \theta \cos \phi \mathbf{i}+\sin \theta \sin \phi \mathbf{j}+\cos \theta \mathbf{k}, \\
\cos \theta^{\prime} & =\mathbf{r} \cdot \mathbf{Z}^{\prime}, \\
& =\cos \xi \cos \theta+\sin \xi \sin \theta \cos \phi .
\end{aligned}
$$

For the case for which the gluon was emitted by the parton in the antiproton, the angular distribution of the decay positron with respect to the $Z^{\prime}$ proton axis (which defines the quarkantiquark axis) is given by the simple expression.

$$
\frac{d \sigma}{d \cos (\theta)}=A\left[\left(1+\cos ^{2} \theta^{\prime}\right)+\frac{B^{\prime}}{A} \cos \theta^{\prime}\right]
$$

(if the gluon was emitted by the proton, then the above distribution is with respect to the direction of the antiproton which defines the quark-antiquark axis). Therefore, the distributions for the proton direction and for the antiproton direction should be added. Each should be weighted by the probability that the quark emitted the gluon $P_{q}\left(x_{1}\right)$, or the probability that the antiquark emitted a gluon, $P_{\bar{q}}\left(x_{2}\right)$. Here $P_{q}\left(x_{1}\right)+P_{\bar{q}}\left(x_{2}\right)=1$. For proton-antiproton collisions, the two probabilities should be equal. For proton-proton collisions, they could be different.

The proton direction is at an angle $\xi$. The antiproton direction angle is $-\xi$. If we plug in the expression for $\cos \theta^{\prime}$ from (A.2) into (A.3), we get

$$
\begin{aligned}
\frac{d \sigma}{d \cos \left(\theta^{\prime}\right)}= & \operatorname{term}_{q}+\text { term }_{\bar{q}} \\
\operatorname{term}_{q}= & A P_{q}\left(x_{1}\right)\left[1+\cos ^{2} \theta \cos ^{2} \xi\right. \\
& +\sin ^{2} \theta \sin ^{2} \xi \cos ^{2} \phi \\
& +2 \cos \xi \cos \theta \sin \xi \sin \theta \cos \phi \\
& +\frac{B^{\prime}}{A} \cos \xi \cos \theta \\
& \left.+\sin _{\xi} \sin \theta \cos \phi\right] \\
\text { term }_{\bar{q}}= & C P_{\bar{q}}\left(x_{2}\right)\left[1+\cos { }^{2} \theta \cos ^{2} \xi\right. \\
& +\sin ^{2} \theta \sin { }^{2} \xi \cos ^{2} \phi \\
& -2 \cos \xi \cos \theta \sin ^{2} \sin \theta \cos \phi \\
& +\frac{B^{\prime}}{A} \cos \xi \cos \theta \\
& \left.-\frac{B^{\prime}}{A} \sin \xi \sin \theta \cos ^{\prime}\right] .
\end{aligned}
$$

Adding term $_{q}$ and term $_{\bar{q}}$ and defining $\Delta P=\left(P_{q}\left(x_{1}\right)-\right.$ $\left.P_{\bar{q}}\left(x_{2}\right)\right) \approx 0$ we get

$$
\begin{aligned}
\frac{d \sigma}{d \cos (\theta)}= & A\left[1+\cos ^{2} \theta \cos ^{2} \xi\right. \\
& +\sin ^{2} \theta \sin ^{2} \xi \cos ^{2} \phi \\
& +\Delta P 2 \cos \xi \cos \theta \sin \xi \sin \theta \cos \phi \\
& +\frac{B^{\prime}}{A} \cos \xi \cos \theta \\
& \left.+\Delta P \frac{B^{\prime}}{A} \sin \xi \sin \theta \cos \phi\right]
\end{aligned}
$$

using trigonometric identities

$$
\sin 2 \theta=2 \sin \theta \cos \theta,
$$


$\cos ^{2} \phi=\frac{1}{2}(1+\cos 2 \phi)$

we rewrite (A.5) as:

$$
\begin{aligned}
\frac{d \sigma}{d \cos (\theta)}= & A\left[1+\cos ^{2} \theta\right. \\
& +\frac{\sin ^{2} \xi}{2}\left(1-3 \cos ^{2} \theta\right) \\
& +\Delta P 2 \cos \xi \sin \xi \sin ^{2} \theta \cos \phi \\
& +\frac{\sin ^{2} \xi}{2} \sin ^{2} \theta \cos ^{2} \phi \\
& +\Delta P \frac{B^{\prime}}{A} \sin \xi \sin \theta \cos \phi \\
& \left.+\frac{B^{\prime}}{A} \cos \xi \cos \theta\right]
\end{aligned}
$$

The general expression for the total differential cross section for dilepton pair production (e.g. Drell-Yan, $Z$ 's or $W$ 's) in proton-antiproton (or proton-proton) collisions is given by:

$$
\begin{aligned}
\sigma= & A\left[\left(1+\cos ^{2} \theta\right)\right. \\
& +\frac{1}{2} A_{0}\left(1-3 \cos ^{2} \theta\right)+A_{1} \sin 2 \theta \cos \phi \\
& +\frac{1}{2} A_{2} \sin ^{2} \theta \cos 2 \phi+A_{3} \sin \theta \cos \phi \\
& +A_{4} \cos \theta+A_{5} \sin ^{2} \theta \sin ^{2} \phi \\
& \left.+A_{6} \sin 2 \theta \sin \phi+A_{7} \sin \theta \sin \phi\right]
\end{aligned}
$$

where $A_{4}=\frac{B}{A}$. Comparing (A.7) to (A.8) we get:

$$
\begin{aligned}
& A_{0}=\sin ^{2} \xi=\frac{P_{T}^{2}}{P_{T}^{2}+M_{\ell \ell}^{2}}, \\
& A_{1}=\Delta P \cos \xi \sin \xi=\Delta P \frac{P_{T} M_{\ell \ell}}{P_{T}^{2}+M_{\ell \ell}^{2}} \approx 0, \\
& A_{2}=\sin ^{2} \xi=\frac{P_{T}^{2}}{P_{T}^{2}+M_{\ell \ell}^{2}}, \\
& A_{3}=\Delta P \frac{B^{\prime}}{A} \sin \xi=\Delta P \frac{B^{\prime}}{A} \frac{P_{T}}{\left(P_{T}^{2}+M_{\ell \ell}^{2}\right)^{1 / 2}} \approx 0, \\
& A_{4}=\frac{B}{A}=\frac{B^{\prime}}{A} \cos \xi=\frac{B^{\prime}}{A} \frac{M_{\ell \ell}}{\left(P_{T}^{2}+M_{\ell \ell}^{2}\right)^{1 / 2}}, \\
& \frac{B}{B^{\prime}}=\frac{M_{\ell \ell}}{\left(P_{T}^{2}+M_{\ell \ell}^{2}\right)^{1 / 2}}, \\
& A_{5}=A_{6}=A_{7}=0 .
\end{aligned}
$$

Where $B$ is the $\cos \theta$ angular coefficient for quarks bound in the proton, and $B^{\prime}$ is the $\cos \theta$ angular coefficient for free quarks. As far as we know, this is the first closed form derivation of the $Q C D$ corrections to the forward backward asymmetry (i.e. the expression above for $A_{4}$ and $\frac{B}{B^{\prime}}$ ). The above perturbative formulae for $A_{0}$ and $A_{2}$ for the annihilation diagram for Drell-Yan production was first derived by Collins in 1979 [21, 22]. The perturbative expressions for $A_{0}, A_{1}$ and $A_{2}$ and $\Delta P$ (but not for $A_{3}$ and $A_{4}$ ) were also derived in a paper by Daniel Boer and Werner Vogelsang in 2006) [21, 22]. The above expressions for $A_{0}$ and $A_{2}$ were shown to hold for the quark-antiquark annihilation diagram for all orders (re-summation) by Berger et al. in 2007 [23, 24].

In conclusion, if the $Z / D Y$ cross section is dominated by quark-antiquark annihilation (as expected) the perturbative calculation gives predictions for $A_{0}\left(=A_{2}\right)$ and $A_{4}$ as the only surviving nonzero angular coefficients. The simple QCD predictions for the angular coefficients are:

$$
\begin{aligned}
h\left(\theta, M_{\ell \ell}, P_{T}\right) & =\frac{1}{2} A_{0}\left(1-3 \cos ^{2} \theta\right), \\
A_{0} & =\sin ^{2} \xi, \\
\sin ^{2} \xi & =\frac{P_{T}^{2}}{P_{T}^{2}+M_{\ell \ell}^{2}}, \\
A_{4} & =\frac{B}{A}=\frac{B^{\prime}}{A} \cos \xi, \\
\frac{B}{B^{\prime}} & =\cos \xi, \\
\cos \xi & =\frac{M_{\ell \ell}}{\left(P_{T}^{2}+M_{\ell \ell}^{2}\right)^{1 / 2}} .
\end{aligned}
$$

The angular distributions predicted by standard Monte Carlo generators such as PHYTIA [16], ResBos re-summation $^{3}$ [25-27] and Vector Boson Production and Decay in Hadron Collisions Resummation $\operatorname{VBP}^{4}$ [28, 29] for $Z$ boson production at the Tevatron are well described by the above formulae.

On average, both QCD correction terms are very small. For Drell-Yan events the average ratio $P_{T} / M_{\ell \ell}$ is about 0.13 (e.g. $12 \mathrm{GeV} P_{T}$ at the $Z$ mass of $90 \mathrm{GeV}$ ). This implies that on average $\left\langle A_{0}\left(P_{T}\right)\right\rangle=0.018$, and $\left\langle\frac{B}{B^{\prime}}\left(P_{T}\right\rangle=0.991\right.$.

\section{A.1 Including the effect of photon radiation by final state leptons}

Here, we derive an approximate formula for including the effect of final state photons. The more exact way is to run

\footnotetext{
${ }^{3}$ Additional details: http://hep.pa.msu.edu/resum. Code: cpy_113009 $\left(\sin ^{2} \theta_{\mathrm{W}}=0.2314\right)$; Grid files: dy_*_tev2_ct66 w321.00 and yk.00. ${ }^{4}$ We used CTEQ6M PDFs and Z-fermion electroweak coupling $\sin ^{2} \theta_{W}=0.232$.
} 
the Monte Carlo, with and without photon radiation and find a correction to the final result.

We assume that final state radiation smears the measured angle of the final state electrons by an $R M S$ angle $\pm \delta$ in the $\theta$ direction, and the same angle $\pm \delta$ in $\phi$. The $\theta$ smearing is equivalent of taking the average of the above expressions with $\xi+\delta$ and $\xi-\delta$. The $\phi$ smearing is equivalent of taking the average of the above expressions with $2 \phi+\delta$ and $2 \phi-\delta$. Therefore, the effect of final state photon radiation can be approximated by:

$$
\begin{aligned}
A_{0}^{\mathrm{rad}}= & \frac{1}{2}\left[\sin ^{2}(\xi+\delta)+\sin ^{2}(\xi-\delta)\right] \\
= & \sin ^{2}(\xi) \cos ^{2} \delta+\cos ^{2} \xi \sin ^{2} \delta, \\
A_{2}^{\mathrm{rad}}= & {\left[\sin ^{2} \xi \cos ^{2} \delta+\cos ^{2} \xi \sin ^{2} \delta\right] } \\
& \times \frac{1}{2}[\cos (2 \phi+\delta)+\cos (2 \phi+\delta)] \\
= & {\left[\sin ^{2} \xi \cos ^{2} \delta+\cos ^{2} \xi \sin ^{2} \delta\right] \cos \delta } \\
= & A_{0}^{\mathrm{rad}} \cos \delta, \\
A_{4}^{\mathrm{rad}=}= & \frac{B^{\prime}}{A} \frac{1}{2}[\cos (\xi+\delta) \cos (\xi-\delta)] \\
= & \frac{B^{\prime}}{A} \cos \xi ! \cos \delta .
\end{aligned}
$$

The above formula approximates the effects of photon radiation using one effective parameter $\delta$. That parameter depends on the specific experimental cuts and channels. For example, if the final state is composed of two electrons, some of the photons are merged in with the electron shower in the calorimeter and help restore part of the original electron energy and direction. If the final state is a dimuon, the radiated photons are not included in the muon energy. Therefore $\delta$ is only an effective parameter that needs to be determined from fits to a Monte Carlo simulation. Probably, the simplest way is to include the corrections for the effect of photon radiation as part of an overall MC radiative correction. Alternatively, we could extract $\delta$ from a fit to the MC and parametrize the $\mathrm{QCD}+\mathrm{QED}$ corrections to the angular distribution by:

$$
\begin{aligned}
h\left(\theta, M_{\ell \ell}, P_{T}\right) & =\frac{1}{2} A_{0}^{\mathrm{rad}}\left(1-3 \cos ^{2} \theta\right), \\
A_{0}^{\mathrm{rad}} & =\sin ^{2} \xi \cos ^{2} \delta+\cos ^{2} \xi \sin ^{2} \delta, \\
A_{4}^{\mathrm{rad}} & =\frac{B^{\mathrm{rad}}}{A}=\frac{B^{\prime}}{A} \cos \xi \cos \delta,
\end{aligned}
$$

$$
\begin{aligned}
\frac{B^{\mathrm{rad}}}{B^{\prime}} & =\cos \xi \cos \delta, \\
\sin ^{2} \xi & =\frac{P_{T}^{2}}{P_{T}^{2}+M_{\ell \ell}^{2}}, \\
\cos \xi & =\frac{M_{\ell \ell}}{\left(P_{T}^{2}+M_{\ell \ell}^{2}\right)^{1 / 2}} .
\end{aligned}
$$

For $\delta$ of order 0.1, the effect of QED photons is of the same magnitude as the effect of the QCD corrections.

\section{References}

1. J.L. Rosner, Phys. Rev. D 35, 2244 (1987)

2. M. Cvetiÿc, S. Godfrey, in Electroweak Symmetry Breaking and New Physics at the TeV Scale, ed. by T.L. Barklow et al. (World Scientific, Singapore, 1995), p. 383. hep-ph/9504216

3. D. London, J.L. Rosner, Phys. Rev. D 34, 1530 (1986)

4. M.S. Carena, A. Daleo, B.A. Dobrescu, T.M.P. Tait, Phys. Rev. D 70, 093009 (2004)

5. M.S. Chanowitz, arXiv:0903.2497 [hep-ph]

6. F. Abe et al. (CDF Collaboration), Phys. Rev. Lett. 79, 2192 (1997)

7. A. Abulencia et al. (CDF Collaboration), Phys. Rev. Lett. 96, 211801 (2006)

8. M. Pillai, Ph.D. Thesis, Univ. of Rochester, UR-1478, 1996

9. B. Abbott et al., Phys. Rev. Lett. 82, 4769 (2000)

10. J. Rosner, Phys. Rev. D 54, 1078 (1996)

11. J. Rosner, Phys. Rev. D 35, 2244 (1987)

12. V. Barger, D.G. Deshpande, J. Rosner, K. Whisnant, Phys. Rev. D 35, 2893 (1987)

13. J. Rosner, Phys. Rev. D 61, 016006 (2000)

14. A. Bodek, U. Baur, Eur. Phys. J. C 21, 607 (2001)

15. J.C. Collins, D.E. Soper, Phys. Rev. D 16, 2219 (1977)

16. T. Sjøstrand et al., J. High Energy Phys. 05, 026 (2006)

17. U. Baur, O. Brein, W. Hollik, C. Schappacher, D. Wackeroth, Phys. Rev. D 65, 033007 (2002)

18. A. Bodek, Nucl. Instrum. Methods. 117, 613 (1974); errata 150, 367 (1978)

19. A. Bodek, SLAC- TN-74-2 (1974, unpublished)

20. J. Han, Ph.D. Thesis, University of Rochester, FERMILABTHESIS-2008-65

21. J.C. Collins, Phys. Rev. Lett. 42, 291 (1979)

22. D. Boer, W. Vogelsang, Phys. Rev. D 74, 014004 (2006)

23. E.L. Berger, J.-W. Qiu, R.A. Rodriguez-Pedraza, Phys. Rev. D 76, 074006 (2007)

24. E.L. Berger, J.-W. Qiu, R.A. Rodriguez-Pedraza, Phys. Lett. B 656 (2007)

25. G.A. Ladinsky, C.-P. Yuan, Phys. Rev. D 50, 4239 (1994)

26. C. Balazs, C.-P. Yuan, Phys. Rev. D 56, 5558 (1997)

27. F. Landry, R. Brock, P.M. Nadolsky, C.-P. Yuan, Phys. Rev. D 67, $073016(2003)$

28. R.K. Ellis, S. Veseli, Nucl. Phys. B 511, 649 (1998)

29. R.K. Ellis, D.A. Ross, S. Veseli, Nucl. Phys. B 503, 309 (1997) 